\title{
Medications used in dementia: a review of evidence
}

\author{
C Suraweera, R Hanwella, VA de Silva
}

\section{Background}

Dementia is an acquired global impairment of intellect, memory and personality, without impairment of consciousness. It is usually progressive in nature. The management of dementia is mainly two faceted; pharmacological and non-pharmacological.

Aims

To review the evidence regarding the efficacy of cholinesterase inhibitors, memantine and antipsychotics, in the treatment of dementia.

\section{Methods}

We searched ALOIS, Cochrane database, PubMed, Scopus and Google scholar using key words; dementia, cognitive enhancers, cholinesterase inhibitors and memantine as individual words for relevant review articles.

Results

There is evidence of efficacy of donepezil, rivastigmine and galantamine in mild to moderate Alzheimer's dementia (AD). Evidence also suggests that memantine has efficacy in moderate to severe $A D$. Further, memantine may benefit behavioural and psychological symptoms of dementia (BPSD), and has also being considered for the treatment of dementias of lesser severity.

Evidence also supports the use of AChE inhibitors in vascular dementia and dementia due to Parkinson's disease (PDD). But evidence regarding efficacy in dementia of Lewy Body (DLB) is not very strong.

Antipsychotics can be used for the management of BPSD if other measures fail, keeping in mind the black box warning for the risk of stroke. The use of antidepressants and mood stabilisers are limited by the lack of strong evidence.

\section{Conclusion}

The current evidence supports the use of cholinesterase inhibitors and memantine in patients with Alzheimer's dementia, vascular dementia and PDD, with a positive impact on global assessment, cognitive function, behavioural disturbance and activities of daily living rating scales. The effect in DLB remains unclear.

SL J Psychiatry 2015; 6(2): 3-8

\section{Introduction}

Dementia is an acquired global impairment of intellect, memory and personality, but without impairment of consciousness. It is usually progressive in nature. The pattern of cognitive impairment depends on the type and severity of dementia. Impairments of cognitive function are commonly accompanied, and occasionally preceded by, deterioration in emotional control, social behaviour, or motivation resulting in significant impairment in activities of daily living (1). The noncognitive symptoms associated with dementia such as mood, psychotic and sleep-wake cycle disturbances i.e., the behavioural and psychological symptoms of dementia (BPSD) - and are seen in about $50-80 \%$ of patients (2).

The two main classification systems used in the world to diagnose psychiatric illness are the Diagnostic and Statistical Manual of Mental Disorders - 5 (DSM-5) and the International Classification of Diseases (ICD-10) (1). The DSM-5, released in 2013, has replaced the word dementia with the term 'major neurocognitive disorder (NCD)' (3). Both the ICD-10 and DSM-5 require impairment in two or more cognitive domains, sufficient to cause difficulties in social and occupational functioning, for the diagnosis of dementia. Screening tests for dementia include the Mini Mental State Examination (MMSE) and the Montreal Cognitive Assessment (MoCA). More definitive tests for diagnostic and monitoring purposes of dementia include the Alzheimer's Disease Assessment Scale (ADAS), Addenbrook's Cognitive Assessment-Revised (ACE-R) and the Repeatable Battery for the Assessment of Neuropsychological Status (RBANS).

Alzheimer's disease (AD) is the commonest type of dementia, accounting for about $50-60 \%$ of all dementias. The prevalence of AD is 1 to $2 \%$ at the age of 65 , but the prevalence doubles every 5 years after that (4). Memory impairment is prominent although it is not the only cognitive domain that is affected (5). Vascular dementia (VaD) accounts for about $20-25 \%$ of all dementias. The management options include controlling of cerebrovascular and metabolic risk factors $(5,6)$. The clinical features of dementia with Lewy bodies (DLB) and Parkinson's disease with dementia (PDD) are similar. The diagnosis of PDD rests on the occurrence of dementia in 
a person formally diagnosed with Parkinson's disease at least 12-months previously. DLB accounts for 15$20 \%$ of cases of dementia. Characteristic symptoms are deterioration of attention and visuospatial abilities, fluctuations in cognition and attention, well-formed visual hallucinations with motor features of Parkinsonism (5).

The management of dementia is mainly two faceted; pharmacological and non-pharmacological. Both these approaches are utilised for the management of the two main symptom domains; cognitive and non-cognitive. Although not strictly disease modifying, cognitive enhancers are used to manage the cognitive symptoms while antipsychotics, antidepressants, benzodiazepines and mood stabilizers are used to treat non-cognitive symptoms.

\section{Methods}

We searched ALOIS (a comprehensive, open-access register of dementia studies), Cochrane database, PubMed, Scopus and Google scholar using key words: dementia, cognitive enhancers, cholinesterase inhibitors and memantine. Our review is based on review articles indexed in these databases and the RCTs included in the reviews. We did not record the number of articles or assess the quality of the articles as this is not a systematic review. Relevance of the articles were determined by careful scrutiny of the contents by all three authors.

\section{Results}

\section{Cognitive enhancers}

The principal class of medication used for the treatment of dementia is cognitive enhancers, which includes cholinesterase (AChE) inhibitors, such as donepezil, rivastigmine, galantamine, and $\mathrm{N}$-Methyl-D-aspartate (NMDA) antagonists, such as memantine.

\section{Acetylcholinesterase (AChE) inhibitors}

The cholinergic hypothesis of $\mathrm{AD}$ attributes cognitive deterioration to progressive loss of cholinergic neurons and decreasing levels of acetylcholine (ACh) in the brain (7). The three AChE inhibitors used in mild to moderate AD are donepezil, rivastigmine and galantamine, of which donepezil and rivastigmine are available in Sri Lanka.

In Alzheimer's disease a meta-analysis of 13 RCTs found that treatment for over 6 months produced improvements in cognitive function, of on average -2.7 points (95\% CI -3.0 to -2.3) on the ADAS-cog scale. Most trials were on patients with mild-moderate dementia. Benefits were also seen on measures of Activities of Daily Living (ADL) and behaviour, with small effect sizes (5). The metaanalyses reported no significant differences in efficacy between different AChE (8-11), a finding that may have been influenced by the small effect sizes.
Although there is a scarcity of comparative data for definitive conclusions to be made, withdrawal rates due to adverse effects for donepezil ranged from 4\%-16\% (placebo 1-7\%), 7\%-29\% for rivastigmine (placebo 7\%) and $7 \%-23 \%$ for galantamine (placebo 7-9\%). The number needed to harm (NNH) was 12 (8).

\section{Donepezil}

A 12-week RCT of 468 patients reported that donepezil use was associated with statistically significant improvements compared to placebo. The mean drugplacebo differences, at end point for the groups receiving $5 \mathrm{mg} / \mathrm{d}$ and $10 \mathrm{mg} / \mathrm{d}$ of donepezil hydrochloride were, respectively, 2.5 and 3.1 units for the ADAS-cog $(11,12)$. Results from a Cochrane review suggest that donepezil results in statistically significant improvements for both 5 and $10 \mathrm{mg} /$ day at 24 weeks compared with placebo on the ADAS-cog, with a 2.01 point and a 2.80 point reduction, respectively (9). A long-term placebo controlled trial of donepezil in 565 patients with mild-tomoderate AD found a small but significant benefit on cognition compared with placebo. This was reflected in a 0.8 point difference in the MMSE score (95\% CI 0.5$1.2 ; \mathrm{P}<0.0001)$ which was replicated in other similar trials $(14,43)$.

\section{Rivastigmine}

Studies for rivastigmine suggest an advantage of 2.6-4.9 points on the ADAS-cog over placebo (13). A Cochrane review found that, high-dose rivastigmine (6-12 mg daily) was associated with a 2-point improvement in cognitive function on the ADAS-cog and a 2.2-point improvement in ADL at 26 weeks over placebo. At lower doses (4 mg daily or lower), the differences were statistically significant for cognitive functions only (14). According to a 6-month, double-blind, placebo controlled RCT, Rivastigmine transdermal patch $(9.5 \mathrm{mg} / 24 \mathrm{~h})$ is as effective as the highest doses of oral formulations (15).

\section{Galantamine}

A 5-month placebo controlled study of 978 patients found that the galantamine-placebo differences on ADAS-cog were 3.3 points for the $16 \mathrm{mg}$ /day group and 3.6 points for the $24 \mathrm{mg} /$ day group ( $\mathrm{p}<0.001$ versus placebo, both doses) (16). A Cochrane review of ten trials found treatment with galantamine was associated with a significantly greater proportion of subjects with improved or unchanged global rating scale rating ( $\mathrm{k}=8$ studies), at all dosing levels except for $8 \mathrm{mg} / \mathrm{d}$ (17). Galantamine is marginally effective in patients with severe $\mathrm{AD}$ with MMSE scores of 5-12 points $(18,32)$.

\section{Memantine}

Memantine is a moderate-affinity, uncompetitive, voltage-dependent NMDA receptor antagonist. A number-needed-to-treat (NNT) analysis of memantine 
showed a NNT of 3-8 (19). It is indicated for the treatment of moderate to severe AD, and has shown significant efficacy in improving symptoms in several large-scale, controlled clinical studies (20-23). Memantine may be effective in delaying worsening of clinical symptoms, and decreasing the emergence of BPSD (24-25). A metaanalysis done in 2007 of six individual phase III studies using a subgroup of patients with moderate to severe AD showed that the drug resulted in a statistically significant benefit in four domains: namely cognitive, functional, global, and behavioural domains. The metaanalysis also highlighted a significant improvement in ADL with memantine, compared to placebo (22). A metaanalysis in 2011 found no significant differences between memantine and placebo on any outcome for patients with mild $\mathrm{AD}$, either on individual trial or when data were combined (ADAS-cog 0.17; P = 0.82) (25). Memantine appears to be well tolerated (27). However, caution is required in hepatic impairment and seizures. The most frequently reported adverse effects in placebo-controlled trials included agitation (7.5\% memantine, $12 \%$ placebo), falls (6.8\% versus $7.1 \%$ ) and dizziness (6.3\% versus $5.7 \%$ ) (28).

\section{Combination treatment}

Of the combinations, AChE inhibitor and memantine combination is the best tolerated, although there is no clear evidence of superior efficacy $(21,29)$. However, there is some evidence that combining memantine with AChE inhibitors may slow cognitive and functional decline compared with mono-therapy or no treatment in the long term (29).

\section{Statins in dementia}

Four RCT have assessed the efficacy of statins in Alzheimer's or probable Alzheimer's dementia. Most patients were already on AChE. Pooled data showed no significant benefit from statin as measured by the ADASCog (mean difference -0.26, 95\% confidence interval (CI) -1.05 to $0.52, \mathrm{p}=0.51$ ) (48).

\section{Vascular dementia}

Three trials with a total of 800 participants have assessed the use of rivastigmine in vascular dementia (49). The largest included 710 participants with vascular dementia (VaD), including those with subcortical and cortical forms of the disorder. Statistically significant improvement in cognitive response was seen with rivastigmine treatment at 24 weeks, but there was no global impression of change, and no improvement of non-cognitive measures. Two other trials, with 1378 participants, have reported statistically significant treatment effects in favour of galantamine compared with placebo in cognition, activities of daily living and behaviour (50). There is evidence for donepezil, rivastigmine and galantamine use in $\mathrm{VaD}$ (29-31). There is also evidence for the use of memantine in $\mathrm{VaD}(6)$.

\section{Parkinson's disease with dementia and dementia with Lewy bodies}

RCTs have assessed the use of cholinesterase inhibitors in both Parkinson's disease with dementia (PDD) and dementia with Lewy bodies (DLB) (33). Three trials have reported cholinesterase inhibitor treatment to be superior to placebo in PDD, as measured by the Clinical Global Impression of Change (CGIC) score of -0.38 , favouring the cholinesterase inhibitors (95\% CI -0.56 to $-0.24, \mathrm{P}<$ $0.0001)$. There was no statistically significant difference in the MMSE between the control and treatment groups for patients with DLB (33). Although there are some concerns about worsening or adverse responses when patients with DLB are exposed to memantine, a recent RCT found it to be mildly beneficial in terms of global clinical status and behavioural symptoms in patients with DLB(26).

\section{Treatment of behavioural and psychological symptoms of dementia}

Second generation antipsychotics (SGA) are as effective as first generation antipsychotics (FGA) for behavioural and psychological symptoms of dementia (BPSD) $(34,40,45,46)$. Reviews and trials support the efficacy of olanzapine, risperidone, quetiapine, aripiprazole and amisulpiride with no significant differences between treatment groups (34-36).

\section{Benzodiazepines}

Although widely used, benzodiazepines are not recommended as it is associated with cognitive decline and falls (37).

\section{Antidepressants}

Evidence suggests that depression, which is present in $30-50 \%$, can be both a cause and consequence of AD. Reviews indicated that antidepressants (mainly SSRIs) not only showed efficacy in treating BPSD, but were also well tolerated $(47,51)$. Two out of five studies of sertraline versus placebo and one study of sertraline versus haloperidol have shown benefit. Five studies have shown that citalopram is of more benefit compared to risperidone.

\section{Mood stabilisers/anticonvulsants}

RCTs of mood stabilisers in BPSD are only available for carbamazepine and valproate, although gabapentin, lamotrigine and topiramate have also been used (38). A literature review of anticonvulsants in BPSD found that although there are benefits, the evidence is insufficient to support routine use (39).

\section{Discussion}

This review examined the evidence regarding the use of medication in the treatment of dementia. The symptoms 
of dementia can be classified into two broad groups namely cognitive and non-cognitive. The review examined the effects of medication on both groups of symptoms. The interpretation of results in trials is difficult due to the factors involving the disease process itself as well as other confounders. Most trials were conducted on patients with mild to moderate dementia with MMSE scores of 10-26, which introduces a bias.

The main question clinicians need answered is 'will medication halt or reverse the process of cognitive decline, and will this result in improved quality of life?' The average annual rate of decline in untreated patients ranges from 6 to 12 points on the ADAS-cog. A 4-point change in the ADAS-cog score is considered clinically meaningful (42).

Patients are classified in to three groups depending on the response: 'non-responders', who continue to decline at the anticipated rate; 'non-decliners', who neither improve significantly nor decline; and 'improvers', who improve to a clinically relevant extent. Data from trials of 6-months duration indicate that of those with AD being treated with AChE inhibitors, 24-34\% will be 'improvers', compared to only $16 \%$ who will be 'improvers' on placebo. Around 55-70\% will be ‘non-decliners’ (40).

It was previously thought that treatment does not improve cognitive functions, merely halts cognitive decline. However recent RCTs indicate that cognitive functions in nearly one third of patients improve with treatment, and more than half are non decliners. Based on this evidence we recommend starting AChE in all patients diagnosed with mild-moderate $\mathrm{AD}$. The duration of most trials is 24 weeks, although there is some evidence of benefit from longer trials. The data also shows that there is little to choose between the differences of AChE in terms of efficacy. So the choice is dependent on ease of dosing, tolerability, cost and availability $(42,44)$.

The patient's cognitive and functional status should be monitored over 6-month intervals, and pharmacologic therapy should ideally be continued until there are no meaningful social interactions and quality of life has irreversibly deteriorated.

With AChE, side effects such as nausea, vomiting, dizziness, insomnia and diarrhoea are due to excess cholinergic stimulation, which most likely occurs at the start of therapy or when the dose is increased. Donepezil appears to a better side effect profile according to clinical trials. However any significant difference between AChE inhibitors is yet to be identified. Gastrointestinal effects appeared to be more common with oral rivastigmine than with other AChE inhibitors, thus requiring slower titration. The cardiac adverse effects should be borne in mind when prescribing AChE inhibitors due to vagotonic effects.

There are other issues that clinicians need to consider; for instance, the treatment response with AChE inhibitors is lost when the medications are interrupted and may not be fully regained when it is reinitiated (42). And failure to benefit from one AChE inhibitor does not necessarily mean that a patient will not respond to another and poor tolerability to one agent does not rule out good tolerability to another (28).

Memantine is a NMDA receptor antagonist, which can be used in Sri Lanka under a personal license. Unlike AChE it is effective in moderate-severe AD. It is effective in treating cognitive decline and behavioural problems and may be better tolerated than AChE by some patients.

Most of the trials have been conducted in patients with Alzheimer's dementia. It is important to know if these medications are effective in other types of dementia too. Vascular dementia is the second most common type of dementia after Alzheimer's disease. In older patients in particular, the combination of vascular dementia and Alzheimer's disease is common, and is referred to as mixed dementia. Evidence supports the use of AChE in vascular dementia $(49,50)$.

The currently available evidence supports the use of cholinesterase inhibitors in patients with PDD, with a positive impact on global assessment, cognitive function, behavioural disturbance and activities of daily living rating scales. The effect in DLB remains unclear (33).

Behavioral and psychological symptoms of dementia include agitation, depression, apathy, repetitive questioning, psychosis, aggression, sleep problems, wandering, and a variety of inappropriate behaviors (41). As dementia progresses treatment of behavioural and psychological symptoms become more difficult. Firstgeneration antipsychotics (FGAs) which have been used for a long time for BPSD are being replaced by the secondgeneration antipsychotics (SGA), as they are better tolerated due to the absence of extra pyramidal side effects (45). However, their use is limited by controversial issues, such as small effect sizes, poor tolerability and possible association with increased mortality $(45,46)$. The reports of increase of stroke have led to a black box warning for the use of SGAs for BPSD. Therefore antipsychotics should be used with caution and for a limited period, where other methods such as behavioural modification have failed.

Recent data supports the efficacy of SSRI in treating depressive symptoms in dementia. Findings also suggest that in treatment of AD patients with cholinesterase inhibitors and SSRIs may offer some degree of protection against the adverse effects of depression on cognition (47). Tricyclic antidepressants are not recommended due to the poor side effects profile.

\section{Conclusion}

The currently available evidence supports the use of cholinesterase inhibitors and memantine in patients with Alzheimer's dementia, vascular dementia and PDD, with a positive impact on global assessment, cognitive function, behavioural disturbance and activities of daily 
living rating scales. Antipsychotics should be used with caution, when needed and when other treatment measures have failed. Antidepressants have shown to be useful in those patients with dementia who also have features of depression.

\section{Suraweera, Leicestershire Partnership NHS Trust, United Kingdom \\ R Hanwella, Department of Psychological Medicine, Faculty of Medicine, University of Colombo \\ VA de Silva, Department of Psychological Medicine, Faculty of Medicine, University of Colombo \\ Corresponding author: C Suraweera \\ Email: dr.chathurie@gmail.com \\ DOI http://dx.doi.org/10.4038/sljpsyc.v6i2.8073}

\section{Declaration of interest}

None declared

\section{References}

1. World Health Orgnization (WHO): The ICD-10 classification of mental and behavioural disorders: Clinical descriptions and diagnostic guidelines. Geneva: WHO; 1992.

2. Lyketsos CG, Lopez O, Jones B, Fitzpatrick AL, Breitner J, DeKosky S. Prevalence of neuropsychiatric symptoms in dementia and mild cognitive impairment: results from the cardiovascular health study. JAMA 2002; 288(12): 1475-83.

3. American Psychiatric Association (AMA). DSM-IV: Diagnostic and statistical manual of mental disorders. 4th ed. Washington, DC: AMA; 1994.

4. Nussbaum RL, Ellis CE. Alzheimer's disease and Parkinson's disease. N Engl J Med 2003; 348(14): 1356-64.

5. Cowen P, Harrison PJ, Burns T, editors. Shorter Oxford textbook of psychiatry. 6th ed. Oxford: Oxford University Press; 2012.

6. Kavirajan H, Schneider LS. Efficacy and adverse effects of cholinesterase inhibitors and memantine in vascular dementia: a meta-analysis of randomised controlled trials. Lancet Neurol 2007; 6(9): 782-92.

7. Francis PT, Palmer AM, Snape M, Wilcock GK. The cholinergic hypothesis of Alzheimer's disease: a review of progress. J Neurol Neurosurg Psychiatry 1999; 66(2): 137-47.

8. Birks J. Cholinesterase inhibitors for Alzheimer's disease. Cochrane Database Syst Rev 2006 (1): CD005593.

9. Lanctot KL, Herrmann N, Yau KK, et al. Efficacy and safety of cholinesterase inhibitors in Alzheimer's disease: a meta-analysis. CMAJ 2003; 169(6): 557-64.

10. Wilcock G, Howe I, Coles H, et al. A long-term comparison of galantamine and donepezil in the treatment of Alzheimer’s disease. Drugs Aging 2003; 20(10): 777-89.
11. Figiel GS, Koumaras B, Meng X, Strigas J, Gunay I. Longterm safety and tolerability of rivastigmine in patients with Alzheimer's disease switched from donepezil: an open-label extension study. Prim Care Companion J Clin Psychiatry 2008; 10(5): 363-7.

12. Rogers SL, Doody RS, Mohs RC, Friedhoff LT. Donepezil improves cognition and global function in Alzheimer disease: a 15-week, double-blind, placebo-controlled study. Donepezil Study Group. Arch Intern Med 1998; 158(9): 1021-31.

13. Rosler M, Anand R, Cicin-Sain A, et al. Efficacy and safety of rivastigmine in patients with Alzheimer's disease: international randomised controlled trial. BMJ 1999; 318(7184): 633-8.

14. Courtney C, Farrell D, Gray R, et al. Long-term donepezil treatment in 565 patients with Alzheimer's disease (AD2000): randomised double-blind trial. Lancet 2004; 363(9427): 2105-15.

15. Winblad B, Cummings J, Andreasen N, et al. A six-month double-blind, randomized, placebo-controlled study of a transdermal patch in Alzheimer's disease--rivastigmine patch versus capsule. Int J Geriatr Psychiatry 2007; 22(5): 456-67.

16. Tariot PN, Solomon PR, Morris JC, Kershaw P, Lilienfeld S, Ding C. A 5-month, randomized, placebo-controlled trial of galantamine in AD. The Galantamine USA-10 Study Group. Neurology 2000; 54(12): 2269-76.

17. Loy C, Schneider L. Galantamine for Alzheimer's disease and mild cognitive impairment. Cochrane Database Syst Rev 2006; (1): CD001747.

18. Burns A, Bernabei R, Bullock R, Cruz Jentoft AJ, Frolich L, Hock C, et al. Safety and efficacy of galantamine (Reminyl) in severe Alzheimer's disease (the SERAD study): a randomised, placebo-controlled, double-blind trial. Lancet Neurol 2009; 8(1): 39-47.

19. Livingston $\mathrm{G}$, Katona $\mathrm{C}$. The place of memantine in the treatment of Alzheimer's disease: a number needed to treat analysis. Int J Geriatr Psychiatry 2004; 19(10): 919-25.

20. Reisberg B, Doody R, Stoffler A, et al. Memantine in moderate-to-severe Alzheimer's disease. N Engl J Med 2003; 348(14): 1333-41.

21. Tariot PN, Farlow MR, Grossberg GT, et al. Memantine treatment in patients with moderate to severe Alzheimer disease already receiving donepezil: a randomized controlled trial. JAMA 2004; 291(3): 317-24.

22. Winblad B, Jones RW, Wirth Y, Stoffler A, Mobius HJ. Memantine in moderate to severe Alzheimer's disease: a meta-analysis of randomised clinical trials. Dement Geriatr Cogn Disord. 2007; 24(1): 20-7.

23. Wilcock GK, Ballard CG, Cooper JA, Loft H. Memantine for agitation/aggression and psychosis in moderately severe to severe Alzheimer's disease: a pooled analysis of 3 studies. J Clin Psychiatry 2008; 69(3): 341-8.

24. Wilkinson D, Andersen HF. Analysis of the effect of memantine in reducing the worsening of clinical symptoms in patients with moderate to severe Alzheimer's disease. Dement Geriatr Cogn Disord 2007; 24(2): 138-45.

25. Schneider LS, Dagerman KS, Higgins JP, McShane R. Lack of evidence for the efficacy of memantine in mild Alzheimer disease. Arch Neurol 2011; 68(8): 991-8. 
26. Emre M, Tsolaki M, Bonuccelli U, et al. Memantine for patients with Parkinson's disease dementia or dementia with Lewy bodies: a randomised, double-blind, placebocontrolled trial. Lancet Neurol 2010; 9(10): 969-77.

27. Parsons CG, Danysz W, Quack G. Memantine is a clinically well tolerated N-methyl-D-aspartate (NMDA) receptor antagonist - a review of preclinical data. Neuropharmacology 1999; 38(6): 735-67.

28. Farlow MR, Graham SM, Alva G. Memantine for the treatment of Alzheimer's disease: tolerability and safety data from clinical trials. Drug Saf 2008; 31(7): 577-85.

29. Atri A, Shaughnessy LW, Locascio JJ, Growdon JH. Longterm course and effectiveness of combination therapy in Alzheimer disease. Alzheimer Dis Assoc Disord 2008; 22(3): 209-21.

30. Roman GC, Wilkinson DG, Doody RS, Black SE, Salloway SP, Schindler RJ. Donepezil in vascular dementia: combined analysis of two large-scale clinical trials. Dement Geriatr Cogn Disord 2005; 20(6): 338-44.

31. Moretti R, Torre P, Antonello RM, et al. Rivastigmine superior to aspirin plus nimodipine in subcortical vascular dementia: an open, 16-month, comparative study. Int J Clin Pract 2004; 58(4): 346-53.

32. Small G, Erkinjuntti T, Kurz A, Lilienfeld S. Galantamine in the treatment of cognitive decline in patients with vascular dementia or Alzheimer's disease with cerebrovascular disease. CNS Drugs 2003; 17(12): 90514.

33. Rolinski M, Fox C, Maidment I, McShane R. Cholinesterase inhibitors for dementia with Lewy bodies, Parkinson's disease dementia and cognitive impairment in Parkinson's disease. Cochrane Database Syst Rev 2012; 3: CD006504.

34. De Deyn PP, Rabheru K, Rasmussen A, et al. A randomized trial of risperidone, placebo, and haloperidol for behavioral symptoms of dementia. Neurology 1999 22; 53(5): 946-55.

35. Rainer M, Haushofer M, Pfolz H, Struhal C, Wick W. Quetiapine versus risperidone in elderly patients with behavioural and psychological symptoms of dementia: efficacy, safety and cognitive function. Eur psychiatry 2007; 22(6): 395-403.

36. Lim HK, Pae CU, Lee C, Lee CU. Amisulpride versus risperidone treatment for behavioral and psychological symptoms in patients with dementia of the Alzheimer type: a randomized, open, prospective study. Neuropsychobiology 2006; 54(4): 247-51.

37. Lagnaoui R, Moore N, Moride Y, Miremont-Salame G, Begaud B. Benzodiazepine utilization patterns in Alzheimer's disease patients. Pharmacoepidemiol Drug Saf 2003; 12(6): 511-5.
38. Lonergan E, Luxenberg J. Valproate preparations for agitation in dementia. The Cochrane Database Syst Rev 2009 (3): CD003945.

39. Konovalov S, Muralee S, Tampi RR. Anticonvulsants for the treatment of behavioral and psychological symptoms of dementia: a literature review. Int Psychogeriatr 2008; 20(2): 293-308.

40. Rosler M, Anand R, Cicin-Sain A, Gauthier S, Agid Y, Dal-Bianco P, et al. Efficacy and safety of rivastigmine in patients with Alzheimer's disease: international randomised controlled trial. BMJ 1999; 318(7184): 633-8.

41. Kales HC, Gitlin LN, Lyketsos CG. Assessment and management of behavioral and psychological symptoms of dementia. BMJ 2015; 350: h369.

42. Stern RG, Mohs RC, Davidson M, et al. A longitudinal study of Alzheimer's disease: measurement, rate, and predictors of cognitive deterioration. Am J Psychiatry 1994; 151(3): 390-6.

43. Burns A, Gauthier S, Perdomo C. Efficacy and safety of donepezil over 3 years: an open-label, multicentre study in patients with Alzheimer's disease. Int J Geriatr Psychiatry 2007; 22(8): 806-12.

44. Farlow MR, Cummings JL. Effective pharmacologic management of Alzheimer's disease. Am J Med 2007; 120(5): 388-97.

45. Chan WC, Lam LC, Choy CN, Leung VP, Li SW, Chiu HF. A double-blind randomised comparison of risperidone and haloperidol in the treatment of behavioural and psychological symptoms in Chinese dementia patients. Int J Geriatr Psychiatry 2001; 16(12): 1156-62.

46. Group BMJ. How safe are antipsychotics in dementia? Drug Ther Bull 2007; 45(11): 81-5.

47. Rozzini L, Chilovi BV, Conti M, Bertoletti E, Zanetti M, Trabucchi M, et al. Efficacy of SSRIs on cognition of Alzheimer's disease patients treated with cholinesterase inhibitors. Int Psychogeriatr 2010; 22(1): 114-9.

48. McGuinness B, Craig D, Bullock R, Malouf R, Passmore P. Statins for the treatment of dementia. Cochrane Database Syst Rev 2014; 7: CD007514.

49. Birks J, McGuinness B, Craig D. Rivastigmine for vascular cognitive impairment. Cochrane Database Syst Rev 2013; 5: CD004744.

50. Birks J, Craig D. Galantamine for vascular cognitive impairment. Cochrane Database Syst Rev 2006; 4: CD004746.

51. Henry G1, Williamson D, Tampi RR. Efficacy and tolerability of antidepressants in the treatment of behavioral and psychological symptoms of dementia, a literature review of evidence. Am J Alzheimers Dis Other Demen 2011; 26(3): 169-83. 